\section{QUALIDADE DE VIDA EM ADOLESCENTES COM NECESSIDADES ESPECIAIS EM UM MUNICÍPIO DE PERNAMBUCO}

\author{
Quality of life in adolescents with special needs in a city of the \\ state of Pernambuco
}
Calidad de Vida de adolescentes con necesidades especiales en un municipio de Pernambuco

\section{RESUMO}

Objetivo: Avaliar a qualidade de vida (QV) e a associação desta com variáveis demográficas de adolescentes com necessidades especiais (física, visual e auditiva). Métodos: Tratou-se de um estudo descritivo, de corte transversal, com 72 adolescentes entre 10 e 19 anos, em instituições de ensino público estadual de Petrolina no estado de Pernambuco, Brasil, no período de agosto a outubro de 2012. Para avaliação da QV, foi utilizado o questionário World Health Organization Quality of Life, versão breve (WHOQOL-BREF). Para análise dos dados, foi utilizada a estatística descritiva e inferencial, aplicando-se os testes MannWhitney e Kruskal-Wallis, com $\mathrm{p}<0,05$. Resultados: Observou-se uma menor percepção da QV global nos deficientes físicos $(57,36 \pm 20,67)$. A QV foi melhor percebida pelos deficientes visuais $(65,62 \pm 22,49)$. No tocante aos domínios, entre os deficientes físicos $(60,41 \pm 7,30)$, visuais $(55,84 \pm 13,34)$ e auditivos $(55,00 \pm 20,33)$, os menores escores apresentados foram os de relação social, respectivamente. Os escolares inseridos em sala especial e os adolescentes mais novos obtiveram uma menor percepção da QV. Conclusão: A QV foi melhor percebida pelos deficientes visuais, e os adolescentes com deficiência física apresentaram-se como o grupo mais vulnerável na percepção da QV global e nos domínios físico e meio ambiente entre os escolares investigados. O grupo mais jovem e os estudantes de sala especial perceberam sua QV inferior quando comparada com os mais velhos e os alunos de sala regular.

Descritores: Qualidade de Vida; Adolescente; Pessoa com Deficiência Física; Pessoas com Deficiência Visual; Deficiência Auditiva.

\section{ABSTRACT}

Objective: To evaluate the quality of life (QOL) and its association with demographic variables of adolescents with special needs (physical, visual, and hearing impairments). Methods: This was a descriptive, cross-sectional study, with 72 adolescents aged 10 to 19 years in state public educational institutions of Petrolina, in the state of Pernambuco, Brazil, in the period from August to October 2012. For QOL evaluation, the World Health Organization Quality of Life, short version instrument (WHOQOL-BREF), was applied. For data analysis, descriptive and inferential statistics were used, applying the Mann-Whitney and Kruskal-Wallis tests, with $p<0.05$. Results: Lower perception of global QOL was observed in those with physical disabilities $(57.36 \pm 20.67)$. The QOV was best perceived by the visually impaired $(65.62 \pm 22.49)$. As regards the domains, the lowest scores were obtained in the social relationship for those with physical disabilities $(60.41 \pm 7.30)$, visual (55.84 \pm 13.34$)$, and hearing (55.00 \pm 20.33$)$ impairments. The students placed in special classes and the youngest adolescents reported lower perceived QOL. Conclusion: Among the assessed students, quality of life was best perceived by the visually impaired, and the adolescents with physical disabilities presented themselves as the most vulnerable group in the perception of global QOL, and in the physical and environment domains as well. The youngest group and the students of special classes room perceived their $Q O L$ to be lower compared to the perceived QOL of older students and the ones placed in general education classes.

Descriptors: Quality of Life; Teenager; Person with Physical Disabilities, People with Visual Impairment, Hearing Impairment.
Artigo Original

\section{Christielle Lidianne Alencar Marinho(1) $^{(1)}$}

Sandra Conceição Maria Vieira ${ }^{(2)}$

1) Universidade de Pernambuco - UPE Petrolina (PE) - Brasil

2) Universidade de Pernambuco - UPE Camaragibe (PE) - Brasil
Recebido em: 26/06/2014 Revisado em: 10/10/2014 Aceito em: 20/12/2014 


\section{RESUMEN}

Objetivo: Evaluar la calidad de vida (CV) y su asociación con las variables demográficas de adolescentes con necesidades especiales (física, visual y auditiva). Métodos: Se trató de un estudio descriptivo, de corte transversal, con 72 adolescentes entre 10 y 19 años de instituciones de enseñanza pública del estado de Petrolina-PE entre agosto y octubre de 2012. Fue utilizado el cuestionario World Health Organization Quality of Life, versión breve (WHOQOL-bref) para la evaluación de la CV. Para el análisis de los datos fue realizada la estadística descriptiva e inferencial con la aplicación de la prueba de MannWhitney y Kruskal-Wallis, con $p<0,05$. Resultados: Se observó baja percepción de la CV global de los deficientes físicos (57,36 $\pm 20,67)$. Los deficientes visuales tuvieron mejor percepción de la CV $(65,62 \pm 22,49)$. Respecto a los dominios entre los deficientes físicos $(60,41 \pm 7,30)$, visuales $(55,84 \pm 13,34)$ y auditivos $(55,00$ $\pm 20,33)$ las menores puntuaciones fueron las de la relación social, respectivamente. Los escolares de clases especiales y los adolescentes más jóvenes presentaron baja percepción de la $\mathrm{CV}$. Conclusión: De los escolares investigados, los deficientes visuales percibieron mejor la $C V$ y los adolescentes con deficiencia física fueron los más vulnerables en la percepción de la CV global y los dominios físicos y medio ambiente. El grupo más joven y los estudiantes de clase especial percibieron que su CV era inferior al compararse a los mayores y los alumnos de clase regular.

Descriptores: Calidad de Vida; Adolescente; Personas con discapacidad; Personas con daño visual; Pérdida Auditiva.

\section{INTRODUÇÃO}

A adolescência é a fase do desenvolvimento humano marcada por transformações biológicas da puberdade e relacionada à maturidade biopsicossocial, sendo uma fase crítica, uma vez que envolve momentos de definições de identidade e valores. Esse período não pode ser considerado independente do contexto social, cultural ou político no qual o indivíduo está inserido e tem bastante influência na qualidade de vida dos adolescentes ${ }^{(1)}$.

Para os adolescentes com deficiência, essa fase tem nuances próprias, pois, além dos conflitos característicos do momento, eles têm que formar sua identidade como pessoas que possuem uma deficiência, e muitas vezes a autonomia de vida adulta não lhes é conferida, uma vez que são percebidos pela sociedade como dependentes de cuidados e proteção, sendo excluídos das oportunidades de acesso aos programas oferecidos para pessoas sem deficiência ${ }^{(2)}$.

Com o aumento da expectativa de vida, o número de pessoas com deficiência está crescendo. A Organização Mundial de Saúde (OMS) estima que cerca de 10\% da população de qualquer país em tempo de paz é portadora de algum tipo de deficiência ${ }^{(3)}$, porém, segundo o censo do Instituto Brasileiro de Geografia e Estatística (IBGE) de $2010^{(4)}$, no Brasil, cerca de 45,5 milhões de pessoas se declaram com algum tipo de deficiência, correspondendo a $23,9 \%$ da população brasileira. Apesar de representarem uma quantidade representativa da população, do ponto de vista social e político, os deficientes ainda são vistos como uma minoria, justificando a escassez de literatura relacionada ao tema ${ }^{(5)}$.

Atualmente, a expressão "qualidade de vida" (QV) está amplamente divulgada na linguagem corrente e na literatura científica, com crescente interesse pelo seu conceito. Ela tem sido alvo de muitas definições ao longo dos anos por jornalistas, políticos profissionais de diversas áreas e gestores ligados às políticas públicas, assim como em diferentes campos do saber: economia, sociologia, educação, medicina, enfermagem, psicologia e saúde. O tema igualmente passou a fazer parte do mercado de bens e serviços, a partir da fundação, em 1995, da Associação Brasileira de Qualidade de Vida e da criação do Instituto Brasileiro de Qualidade de Vida (IBQV), instituições sem fins lucrativos que, segundo anunciam, visam à defesa, preservação e conservação do meio ambiente, da ética, da paz, da cidadania, dos direitos humanos e dos valores universais ${ }^{(6)}$.

A OMS, por sua vez, define qualidade de vida como a percepção de um indivíduo em relação à sua posição na vida, no contexto de sua cultura e valores, em relação aos seus objetivos, expectativas e padrões, ainda podendo ser influenciada pela saúde física, pelo estado psicológico, pelo nível de independência, pelas relações sociais, pelos fatores ambientais e pelas crenças pessoais do indivíduo ${ }^{(7)}$.

Apesar de QV ser um conceito subjetivo, para fins de pesquisa, foram desenvolvidos instrumentos que a mensurassem de forma mais objetiva ${ }^{(8)}$. Como exemplo, tem-se o World Health Organization Quality of Life, versão breve (WHOQOL-BREF), proposto pela OMS em $1995^{(7)}$, cuja versão em português foi validada ${ }^{(9)}$. Esse item tem sido utilizado para conhecer a qualidade de vida em diversas populações mundiais, incluindo adolescentes. No entanto, há uma carência de estudos caracterizando a QV em adolescentes com deficiência.

A despeito do avanço alcançado pela educação e pela saúde nas últimas décadas, ainda há muito a ser atingido quanto ao atendimento integral aos adolescentes com deficiência, já que eles necessitam de assistência diferenciada para vivenciarem as mudanças dentro de suas limitações impostas $^{(10)}$. É preciso assegurar que o sistema geral da sociedade - o meio físico e cultural, habitação, transporte, serviços sociais e de saúde, oportunidade de educação e trabalho, vida cultural e social - seja acessível a eles, para a obtenção de uma QV satisfatória. Partindo dessa premissa, é fundamental a discussão e reflexão da QV dos adolescentes 
com deficiência, para que se possa intervir no tocante à emancipação deles e colaborar para uma adolescência mais saudável. Assim, o objetivo do estudo foi avaliar a QV e a associação desta com variáveis demográficas de adolescentes com necessidades especiais (físicas, visuais e auditivas).

\section{MÉTODOS}

Este foi um estudo transversal, descritivo e exploratório, realizado em alunos da rede estadual de ensino da cidade de Petrolina-PE, no período de agosto a outubro de 2012.

A população foi composta por adolescentes com idade entre 10 e 19 anos, matriculados e frequentando regularmente as 22 instituições de ensino estadual de Petrolina-PE que possuem ensino especial. Destas, 18 se localizam na zona urbana e 4 na zona rural. De acordo com o censo 2010 da Secretaria Estadual de Educação (SEDUC$\mathrm{PE})^{(11)}$, existiam 180 alunos com deficiência visual, auditiva e física matriculados.

O tamanho mínimo da amostra foi calculado considerando um erro amostral de 5\%, com um intervalo de $95 \%$ de confiança (IC95\%), desvio padrão da variável de interesse baseada no estudo piloto em 24,16 e mais $20 \%$ em função das possíveis perdas no processo de coleta. A partir desse cálculo, chegou-se a uma amostra de 72 sujeitos, sendo 56 deficientes auditivos, 8 visuais e 8 físicos. A quantidade da amostra por deficiência foi proporcional ao censo da SEDUC ${ }^{(1)}$. Foram incluídos no estudo os adolescentes com deficiência física, visual e auditiva, matriculados em salas regulares e especiais, que estavam frequentando as aulas no período de coleta do estudo. Foram excluídos da pesquisa os adolescentes com deficiência mental, múltipla e aqueles que não conseguiram compreender o instrumento aplicado.

Os alunos foram selecionados por amostragem aleatória simples, considerando a proporcionalidade entre as matrículas dos alunos com deficiência física, visual e auditiva, fornecidas pela Secretária de Educação do Estado de Pernambuco (SEDUC/PE) ${ }^{(11)}$. Para substituição do aluno selecionado que não estivesse na escola no momento da coleta ou que se negasse a participar, procedia-se a um novo sorteio na relação de alunos. Optou-se por essa estratégia de reposição para minimizar as perdas amostrais em determinadas escolas com pequena quantidade de alunos, o que poderia inviabilizar o estudo pela falta de representatividade.

Para a coleta dos dados, foram utilizados dois instrumentos: um questionário sociodemográfico ${ }^{(12)}$ visando à caracterização da amostra e o instrumento para a avaliação da QV da OMS, o WHOQOL-BREF ${ }^{(5)}$. Este instrumento é constituído por 26 questões em escala likert de cinco pontos e considera os últimos 15 dias vividos pelo respondente. Duas questões são referentes à percepção individual da qualidade de vida; as demais estão subdivididas em quatro domínios (físico, psicológico, relações sociais e meio ambiente) e representam cada uma das 24 facetas. Sua versão em português foi validada e adaptada para idioma e cultura brasileira ${ }^{(5)}$.

O instrumento é autoexplicativo e pode ser autoadministrado, assistido pelo entrevistador ou, ainda, administrado pelo entrevistador ${ }^{(5)}$. Neste estudo, a aplicação foi diferenciada, obedecendo aos limites de cada deficiência. Assim, nos deficientes visuais e físicos, foram aplicados em forma de entrevista conduzida pelos pesquisadores, previamente treinados. Nos deficientes auditivos, foi autoaplicado, com auxílio de um intérprete em Libras da própria instituição de ensino, para manter o diálogo entre os pesquisadores e os alunos participantes.

Antes da aplicação deste instrumento, foi realizado um estudo preliminar, com o objetivo de conhecer a população e o instrumento, além de conhecer as possíveis dificuldades oriundas da pesquisa, a fim de minimizá-las no estudo final.

Os dados obtidos no WHOQOL-Bref foram pontuados utilizando-se o programa estatístico Statistical Package for the Social Sciences (SPSS), versão 20.0, conforme sugerido pela $\mathrm{OMS}^{(13)}$. Em um primeiro momento, recorreuse à análise descritiva para a caracterização da amostra, utilizando-se frequências absolutas e relativas, bem como as medidas de tendência central apresentadas por mediana e amplitude interquartil. Em um segundo momento, para comparar os resultados obtidos da qualidade de vida percebida em cada um dos domínios e as características sociodemográficas entre os grupos ou categorias das variáveis, foram utilizados os testes estatísticos inferenciais não paramétricos Mann-Whitney U e Kruskal-Wallis, com um nível de significância igual a 5\%. A justificativa para a escolha dos referidos testes foi a não comprovação da hipótese de normalidade dos dados e os reduzidos tamanhos amostrais $^{(14)}$.

Cumprindo a Resolução no 466/12 do Ministério da Saúde, que versa sobre pesquisa envolvendo seres humanos no Brasil, o presente estudo foi submetido e aprovado no Comitê de Ética da Universidade de Pernambuco, sob o processo $n^{\circ} 150 / 11$. Todos os participantes foram esclarecidos sobre os objetivos da pesquisa e assinaram o Termo de Consentimento Livre e Esclarecido (TCLE). No caso de menores de idade, o TCLE foi assinado pelo responsável.

\section{RESULTADOS}

A amostra foi composta por 72 adolescentes, dos quais $72,2 \%(n=52)$ se enquadravam na faixa de 15 a 19 anos. Considerando a origem das deficiências, percebeu-se que a maior parte delas $-73,6 \%(\mathrm{n}=53)$ - é de origem congênita. Quanto ao sexo, encontrou-se 54,2\% $(\mathrm{n}=39)$ do sexo 
masculino. No tocante à escolaridade, a maioria dos sujeitos - 73,2\% ( $\mathrm{n}=52)$ - estava cursando o ensino fundamental II e $77,8 \%(n=56)$ se encontravam inseridos em sala de ensino regular, ou seja, junto com outros adolescentes sem deficiência (Tabela I).

$\mathrm{Na}$ análise descritiva da QV global, envolvendo toda a amostra, sem diferenciar as deficiências, encontrou-se uma mediana de 63,28. Dentre os domínios, o social apresentou menor escore, seguido do domínio meio ambiente, enquanto o físico se destacou com melhor escore (Tabela II).

Com relação aos domínios da $\mathrm{QV}$, tanto entre os deficientes auditivos quanto entre os deficientes visuais, os menores escores apresentados foram nas relações sociais, diferente dos deficientes físicos, que apresentaram menores escores no meio ambiente. Os maiores escores percebidos entre os deficientes físicos e visuais foram no domínio psicológico, tendo o grupo de deficientes auditivos o melhor escore no domínio físico (Tabela III).

Com base na comparação da QV geral e em algumas características sociodemográficas de todos os adolescentes da amostra, verificou-se que os de faixa etária mais avançada (15 a 19 anos) têm uma melhor percepção da sua QV em relação aos de idade menos avançada $(\mathrm{p}=0,05)$.

No tocante aos domínios, ainda em comparação com as características gerais dos sujeitos, foram encontradas diferenças estatísticas nos domínios social $(\mathrm{p}=0,025)$ e físico $(\mathrm{p}=0,046)$ quando comparados com a idade - os mais velhos percebem melhor a $\mathrm{QV}$ nos dois domínios citados. Com relação ao gênero, houve uma menor percepção da QV nas mulheres, nos domínios físico, psicológico e

Tabela I - Características gerais dos adolescentes da amostra selecionada. Petrolina-PE, 2012.

\begin{tabular}{|c|c|c|c|c|}
\hline \multirow{2}{*}{ Variáveis } & \multicolumn{3}{|c|}{ Deficiência } & \multirow{2}{*}{ Todos } \\
\hline & Física(n=8) & Visual $(n=8)$ & $\operatorname{Auditiva}(n=56)$ & \\
\hline \multicolumn{5}{|l|}{ Idade } \\
\hline 10 a $14 \operatorname{anos}(\%)$ & $4(50,0)$ & $3(37,5)$ & $13(23,2)$ & $20(27,8)$ \\
\hline 15 a $19 \operatorname{anos}(\%)$ & $4(50,0)$ & $5(62,5)$ & $43(76,8)$ & $52(72,2)$ \\
\hline \multicolumn{5}{|l|}{ Gênero } \\
\hline Masculino (\%) & $4(50,0)$ & $4(50,0)$ & $31(55,4)$ & $39(54,2)$ \\
\hline Feminino $(\%)$ & $4(50,0)$ & $4(50,0)$ & $25(44,6)$ & $33(45,8)$ \\
\hline \multicolumn{5}{|l|}{ Origem da deficiência } \\
\hline Adquirida & $4(50,0)$ & $1(12,5)$ & $14(25,0)$ & $19(26,4)$ \\
\hline $\begin{array}{l}\text { Congênita } \\
\text { Renda }\end{array}$ & $4(50,0)$ & $7(87,5)$ & $42(75,0)$ & $53(73,6)$ \\
\hline Até dois salários (\%) & $4(50,0)$ & $1(12,5)$ & $22(43,1)$ & $27(40,3)$ \\
\hline Três ou mais salários (\%) & $4(50,0)$ & $7(87,5)$ & $29(56,9)$ & $40(59,7)$ \\
\hline \multicolumn{5}{|l|}{ Sala de aula } \\
\hline Regular (\%) & $8(100,0)$ & $8(100,0)$ & $40(71,4)$ & $56(77,8)$ \\
\hline Especial (\%) & - & - & $18(28,6)$ & $16(22,2)$ \\
\hline \multicolumn{5}{|l|}{ Região da residência } \\
\hline Urbana (\%) & $6(75,00)$ & $6(75,00)$ & $54(96,40)$ & $66(91,70)$ \\
\hline Rural (\%) & $2(25,00)$ & $2(25,00)$ & $2(3,60)$ & $6(8,30)$ \\
\hline \multicolumn{5}{|l|}{ Escolaridade } \\
\hline Até Fundamental II (\%) & $6(75,00)$ & $6(75,00)$ & $40(72,70)$ & $52(73,20)$ \\
\hline Ensino médio (\%) & $2(25,00)$ & $2(25,00)$ & $15(27,30)$ & $19(26,80)$ \\
\hline
\end{tabular}

Tabela II - Análise descritiva da qualidade de vida de todos os adolescentes com deficiência. Petrolina-PE, 2012.

\begin{tabular}{lcccc}
\hline & \multicolumn{4}{c}{ Qualidade de vida } \\
\cline { 2 - 5 } & $\mathbf{1}^{\mathbf{0}}$ Quartil & $\mathbf{2}^{\mathbf{0}}$ Quartil & $\mathbf{3}^{\mathbf{0}}$ Quartil & $\mathbf{4}^{\mathbf{0}}$ Quartil \\
\hline Domínio Físico & 60,71 & 71,43 & 78,57 & 100,00 \\
Domínio Psicológico & 65,20 & 66,67 & 79,17 & 100,00 \\
Domínio Social & 41,67 & 58,33 & 66,67 & 100,00 \\
Domínio Meio Ambiente & 53,13 & 59,38 & 68,75 & 98,88 \\
Global & 57,30 & 63,28 & 69,07 & 99,22 \\
\hline
\end{tabular}


Tabela III - Qualidade de vida global e domínios do instrumento de avaliação abreviado da qualidade de vida da organização mundial de saúde dos adolescentes com deficiência física, visual e auditiva. Petrolina-PE, 2012.

\begin{tabular}{lcccc}
\hline \multirow{2}{*}{ Domínios } & \multicolumn{4}{c}{ Deficiências } \\
\cline { 2 - 5 } & Física & Visual & Auditiva & $\boldsymbol{p}$ \\
\hline Físico & $56,92 \pm 24,16$ & $68,38 \pm 17,81$ & $70,28 \pm 20,37$ & $0,034^{*}$ \\
Psicológico & $62,50 \pm 43,75$ & $75,00 \pm 29,17$ & $63,67 \pm 14,58$ & 0,098 \\
Social & $60,41 \pm 7,30$ & $55,84 \pm 13,34$ & $55,00 \pm 20,33$ & 0,210 \\
Meio Ambiente & $49,62 \pm 17,93$ & $58,25 \pm 10,94$ & $56,25 \pm 21,87$ & $0,014^{*}$ \\
QV global & $57,36 \pm 20,67$ & $65,62 \pm 22,49$ & $63,82 \pm 18,62$ & $0,036^{*}$ \\
\hline
\end{tabular}

*Diferente estatisticamente dos adolescentes com deficiência física, através do teste Kruskal Wallis.

Tabela IV - Comparação dos fatores sociodemográficos na qualidade de vida de todos os adolescentes com deficiência. Petrolina-PE, 2012.

\begin{tabular}{lccccc}
\hline Variáveis & \multicolumn{5}{c}{ Domínios } \\
\cline { 2 - 6 }$(\%)$ & Físico & Psicológico & Social & Ambiente & Geral \\
\hline Idade & & & & & \\
10 a 14 anos & $67,86 \pm 16,07$ & $64,58 \pm 11,46$ & $50,00 \pm 16,67$ & $54,69 \pm 24,22$ & $57,53 \pm 12,42$ \\
15 a 19 anos & $73,21 \pm 14,29$ & $66,67 \pm 20,83$ & $58,33 \pm 29,17$ & $62,50 \pm 14,84$ & $65,48 \pm 12,22$ \\
Valor de p & $0,046^{*}$ & 0,304 & $0,025^{*}$ & 0,053 & $0,005^{*}$ \\
Sexo & & & & $65,91 \pm 10,46$ \\
Masculino & $75,00 \pm 17,86$ & $66,67 \pm 16,67$ & $50,00 \pm 25,00$ & $65,63 \pm 12,50$ & $62,91,17 \pm 14,24$ \\
Feminino & $71,43 \pm 14,29$ & $62,50 \pm 20,83$ & $58,33 \pm 20,83$ & $56,25 \pm 17,19$ & 64,17 \\
Valor de p & 0,543 & 0,121 & 0,344 & 0,058 & 0,619 \\
Renda & & & & \\
Até dois salários & $71,43 \pm 14,29$ & $70,83 \pm 16,67$ & $58,33 \pm 25,00$ & $65,63 \pm 15,63$ & $65,35 \pm 11,98$ \\
Três ou mais salários & $69,64 \pm 14,39$ & $62,50 \pm 14,58$ & $54,17 \pm 25,00$ & $56,25 \pm 17,97$ & $61,33 \pm 12,57$ \\
Valor de p & 0,258 & 0,094 & 0,882 & $0,035^{*}$ & 0,114 \\
Região da residência & & & & \\
Urbana & $71,43 \pm 17,86$ & $66,67 \pm 16,6$ & $54,17 \pm 25,00$ & $60,94 \pm 15,63$ & $62,95 \pm 12,54$ \\
Rural & $66,07 \pm 12,50$ & $64,58 \pm 9,37$ & $70,83 \pm 20,83$ & $56,25 \pm 17,97$ & $66,29 \pm 11,34$ \\
Valor de p & 0,122 & 0,551 & 0,069 & 0,474 & 0,783 \\
Escolaridade & & & & & \\
Até Fundamental II & $71,43 \pm 17,86$ & $66,67 \pm 16,67$ & $50,00 \pm 25,00$ & $59,38 \pm 15,63$ & $62,52 \pm 13,00$ \\
Ensino médio & $71,43 \pm 7,14$ & $66,67 \pm 8,33$ & $58,33 \pm 25,00$ & $62,50 \pm 12,50$ & $66,30 \pm 11,61$ \\
Valor de p & 0,845 & 0,906 & 0,072 & 0,279 & 0,137 \\
\hline
\end{tabular}

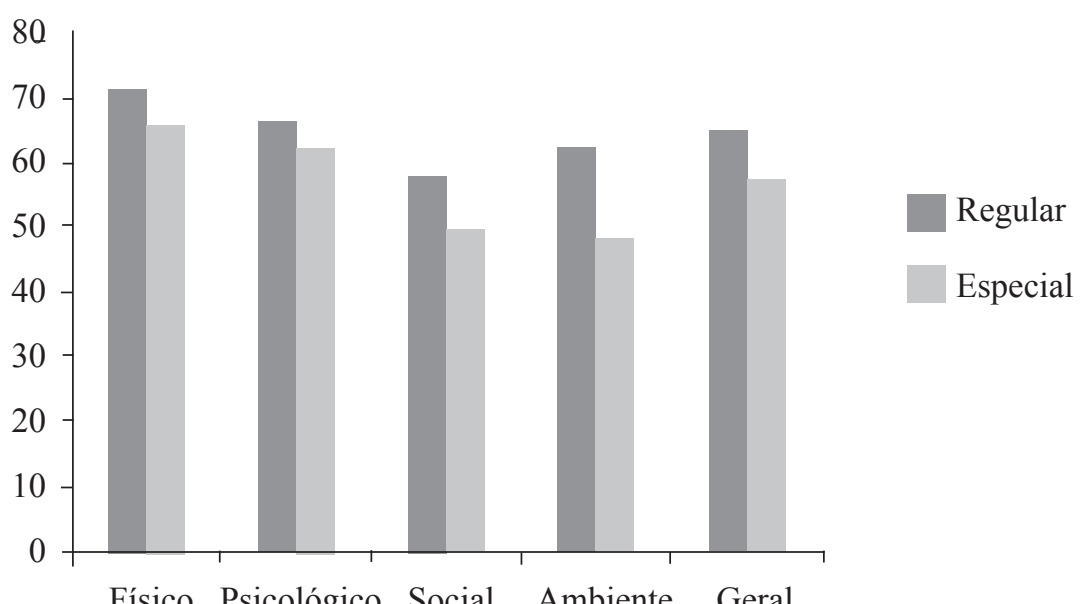

Figura 1 - Comparação dos domínios da qualidade de vida e o tipo de sala de aula dos adolescentes com deficiência auditiva. Petrolina-PE, 2012. 
meio ambiente, embora sem diferença estatisticamente significante. Apenas o domínio relação social apresentou escore superior nas mulheres em detrimento aos homens (Tabela IV).

A variável de inclusão em sala regular e especial foi analisada apenas para os deficientes auditivos, pois é a única deficiência em que ainda existe sala especial no ensino estadual de Petrolina-PE. Assim, foi percebido que tanto a QV global $(p=0,025)$ como todos os domínios se apresentam com melhor resultado nos adolescentes inseridos em salas regulares, junto com outros adolescentes sem deficiência. Os domínios físico $(p=0,027)$ e meio ambiente $(p=0,001)$ apresentaram diferenças significativas (Figura 1).

\section{DISCUSSÃO}

$\mathrm{O}$ interesse pelo estudo da qualidade de vida tem sido crescente em várias áreas da atividade humana. Apesar de não haver consenso quanto à definição de $\mathrm{QV}$, a maioria dos autores concorda que, em sua avaliação, devem ser contemplados os domínios físico, social, psicológico e espiritual, buscando-se captar a experiência pessoal de cada indivíduo ${ }^{(9)}$.

Neste estudo, foi percebido que o domínio "relações sociais" foi o mais acometido na ótica do adolescente com deficiência, seguido do "meio ambiente". O mesmo ocorreu em estudo conduzido na Alemanha, que teve como objetivo verificar a qualidade de vida em 26 adolescentes com autismo, no qual foi encontrado um pior desempenho dos avaliados no domínio das relações sociais e o melhor desempenho foi para o domínio físico ${ }^{(15)}$.

O relacionamento social é uma característica muito comum na adolescência, já que nessa fase existe a tendência grupal. Os adolescentes se identificam com outros jovens, passam a andar juntos e até com as mesmas roupas, formando um grupo com identificação e características próprias - alguns são facilmente identificáveis pelos usos e $\operatorname{costumes}^{(16,17)}$. Para os adolescentes com alguma deficiência, essa característica fica um pouco distorcida, pois muitas vezes eles não conseguem se reconhecer dentro do grupo, por serem diferentes, gerando certo distanciamento dos outros jovens, por medo de serem rejeitados ${ }^{(17)}$.

O domínio meio ambiente foi o segundo mais afetado, porém, na maioria dos estudos nacionais e internacionais utilizando o WHOQOL-Bref. Esse é o domínio mais prejudicado da QV envolvendo adolescentes de diferentes nacionalidades ${ }^{(18-22)}$. Ele engloba aspectos como segurança física e proteção, ambiente no lar, recursos financeiros, cuidados de saúde e sociais, oportunidades de adquirir informações e habilidades, participação em recreação/ lazer, ambiente físico e transporte, sendo alguns desses aspectos não controlados individualmente, dependendo de investimentos governamentais, o que dificulta sua melhora ${ }^{(22)}$.

Com relação à análise dos domínios e percepção global da QV na população em estudo, observaram-se escores significantemente mais baixos entre os adolescentes com deficiência física nos domínios meio ambiente e físico, além da QV global, quando comparados aos deficientes visuais e auditivos. Em alguns estudos com adolescentes que não possuíam deficiência, percebeu-se que os escores do domínio físico são os mais elevados, diferente do atual estudo, que envolveu adolescentes que apresentavam limitações físicas. Esses estudos também indicam que o domínio físico é um contribuinte importante para a $\mathrm{QV}$ positiva entre jovens ${ }^{(23,24)}$. Como esse domínio engloba itens como presença de dor ou desconforto, dependência de medicação, satisfação com o sono, capacidade para o trabalho e atividades diárias, entre outros, espera-se que os escores encontrados sofram alguma interferência decorrente do fato de esses adolescentes possuírem um tipo de deficiência. As limitações primárias impostas pela deficiência aumentam a sua vulnerabilidade para efeitos secundários, sendo a sobrevivência, a saúde e o bem-estar desses jovens dependentes da interação de muitos fatores, incluindo o envolvimento físico e social ${ }^{(24)}$.

A idade tem influência na qualidade de vida - a faixa etária mais velha se mostrou mais satisfeita com sua vida. $\mathrm{O}$ adolescente vive várias perdas e conquistas na transição da identidade infantil para a adulta, influenciando na consolidação da estrutura básica da personalidade. Com o passar dos anos, essas mudanças vão se consolidando e o adolescente adquire certa maturidade, com aquisição de um sistema de valores pessoais e da própria identidade, favorecendo uma melhor $\mathrm{QV}^{(25,26)}$.

Os resultados revelaram, ainda, que os adolescentes do gênero feminino apresentaram uma menor percepção da QV para os domínios psicológico, físico e meio ambiente, quando comparados com os adolescentes do gênero masculino. Em estudo realizado no Paraná ${ }^{(19)}$ analisando o domínio meio ambiente da QV de 608 adolescentes escolares, foi constatado como ponto vulnerável o grupo dos adolescentes do gênero feminino, uma vez que estes possuem maior tendência a problemas psicológicos, como depressão e ansiedade, além de possuírem menor resistência física. Um estudo no Kuwait com 4.467 adolescentes nas escolas constatou que as meninas possuem menor percepção da QV no domínio psicológico, sugerindo que programas escolares em ambientes de estudo amigáveis podem ajudar a promover uma melhor percepção no domínio psicológico, diminuindo os sintomas de ansiedade e depressão comuns entre as meninas ${ }^{(27)}$.

Percebeu-se que a maioria dos alunos com deficiência pesquisados estava inserida em salas com alunos que não 
possuíam deficiências, ou seja, salas regulares. A inclusão dos alunos com deficiência em salas regulares é importante porque lhes permite conviver com outros estudantes, estabelecendo trocas para o desenvolvimento de ambos, como pôde ser percebido neste estudo, cujos indivíduos apresentaram maiores escores tanto na QV geral como em todos os domínios, o que também foi visto em outros estudos $^{(28-30)}$.

A efetivação da inclusão educacional requer clareza sobre a própria condição da pessoa com deficiência, suas habilidades e fragilidades, e neste estudo, propõe-se um olhar para outros aspectos que estão envolvidos na qualidade de vida das pessoas com deficiência, tirando o foco das alterações das funções e estruturas do corpo e pensando os fatores ambientais e pessoais que influenciam a realização de atividades e participação, e permitem a funcionalidade. A escola, sendo uma instituição voltada para a informação e formação, consiste no meio coletivo mais eficaz e favorável à construção de um ambiente saudável, acolhedor, onde todos fazem parte do mesmo processo educacional a partir do respeito às individualidades e como um membro valorizado, pois a educação se processa através do contato e da troca de experiências ${ }^{(31)}$.

Este estudo apresenta algumas limitações que devem ser consideradas, como o número de participantes da pesquisa, que foram subdivididos em grupos menores para a análise dos resultados, e esse fato pode ter levado a não constatação de diferenças estatísticas significantes em alguns domínios avaliados, mas que não tira o valor das diferenças entre os grupos ${ }^{(14)}$. Outra limitação é avaliar exclusivamente por meio de quantificações um conceito intrinsecamente marcado pela subjetividade, como o construto QV. É preciso ter em mente que os indicadores e índices medem sempre "aspectos" da QV. Nesse sentido, os resultados observados no presente estudo precisam ser analisados com cautela, tendo em vista que a medida "objetiva" da QV foi mensurada de forma subjetiva através de questionário. No entanto, é importante ressaltar que o instrumento utilizado tem se apresentado como uma possibilidade de análise da QV prática e confiável - o WHOQOL-Bref é recomendado pela OMS para avaliação da QV.

Outra limitação do presente estudo consiste no fato de que a amostra foi selecionada em escolas públicas estaduais de Petrolina-PE, assim, a extrapolação dos dados para adolescentes que frequentam outras instituições de ensino é limitada. Por fim, este estudo apresenta delineamento transversal e não é possível estabelecer uma relação de causa e efeito entre as deficiências e a QV dos adolescentes.

\section{CONCLUSÃO}

A QV foi mais bem percebida pelos deficientes visuais, e os adolescentes com deficiência física apresentaram-se como o grupo mais vulnerável na percepção da QV global, nos domínios físico e meio ambiente entre os escolares investigados. O domínio relação social foi o mais afetado, quando consideradas todas as deficiências. O grupo mais jovem e os estudantes de sala especial perceberam sua QV inferior em relação aos mais velhos e alunos de sala regular.

Sugere-se a realização de novos estudos dentro dessa temática, abordando aspectos que não foram percebidos ou estudados nesta pesquisa, uma vez que esta população muitas vezes é negligenciada, não possuindo o direito de expressar sua percepção de qualidade de vida.

\section{REFERÊNCIAS}

1. Santos MFS, Félix LB, Morais ERC. Representações Sociais de Juventude em uma Comunidade Quilombola do Agreste Pernambucano. Psico. 2012;43(4):524-32.

2. Seron $\mathrm{BB}$, Interdonato $\mathrm{GC}$, Luiz Junior $\mathrm{CC}$, Greguol M. Prática de atividade física habitual entre adolescentes com deficiência visual. Rev Bras Educ Fís Esp. 2012;26(2):231-39.

3. Organização Mundial da Saúde - OMS. Classificação Internacional de Incapacidades, Funcionalidade e Saúde. Lisboa: OMS; 2004.

4. Instituto Brasileiro de Geografia e Estatística - IBGE. Censo demográfico de 2010 [acesso em 2012 Nov 7]. Disponível em: http://www.censo2010.ibge.gov.br/ resultados_do_censo2010.php

5. Castro SS, Lefèvre F, Lefèvre AMC, César CLG. Acessibilidade aos serviços de saúde por pessoas com deficiência. Rev Saúde Pública. 2011;45(1):99-105.

6. Minayo MCS. Qualidade de vida e saúde como valor existencial. Ciênc Saúde Coletiva. 2013;18(7):1868.

7. The WHOQOL Group. The World Health Organization Quality of Life Assessment (WHOQOL): position paper from the World Health Organization. Soc Sci Med. 1995;41(10):1403-9.

8. Santos JB, Mendonça M, Pinheiro MC, Tamai S, UchidaR, Miorin LA, et al. Negative correlations between anxiety depressive symptoms and quality of life among patients on hemodialysis. São Paulo Med J. 2010;128(2):102-3.

9. Fleck MPA, Louzada S, Xavier M, Chachamovich E, Vieira G, Santos L, Pinzon V. Aplicação da versão em português do instrumento abreviado de avaliação da qualidade de vida "WHOQOL-bref". Rev Saúde Pública. 2000;34(2):178-83.

10. Carvalho RE. Educação inclusiva e a criação de vínculos: um novo olhar. Fed Nac Apaes. 2013;1(1):19-32. 
11. Secretaria de Educação do Estado de Pernambuco. Censo escolar de 2010 [acesso em 2011 Out 15]. Disponível em: http://www.educacao.pe.gov.br

12. Aragão AKR, Souza A, Silva K, Vieira S, Colares, V. Acessibilidade da criança e do adolescente com deficiência na atenção básica de saúde bucal do serviço público: um estudo piloto. Pesq Bras Odontoped Clin Integr. 2011;11(2):159-64.

13. The Whoqol Group. Development of the World Health Organization WHOQOL-bref. Quality of Life Assesment 1998. Psychol Med. 1998;28(3):551-8.

14. Loureiro LMJ, Gameiro MGH. Interpretação crítica dos resultados estatísticos: para lá da significância estatística. Rev Enferm Referência. 2011;3(3):151-62.

15. Kamp-Becker I, Schröder J, Remschmidt H, Bachmann CJ. Health-related quality of life in adolescents and young adults with high functioning autism-spectrum disorder. Psychosoc Med. 2010;7:1-10.

16. Carvalho JN, Erdmann AL, Santana ML. A autonomia do cuidado exercido por adolescentes para um viver saudável: o olhar da enfermagem. Cogitare Enferm. 2011;16(2):268-74.

17. Leite, GA, Monteiro, MIB. A construção da identidade de sujeitos deficientes no grupo terapêutico-fonoaudiológico. Rev Bras Educ Espec. 2008;14(2):189-200.

18. Awasthi S, Agnihotri K, Chandra H, Singh U, Thakur S. Assessment of Health-Related Quality of Life in school-going adolescents: validation of PedsQL instrument and comparison with WHOQOL-BREF. [BVS]. Natl Med J India. 2012;25(2):74-9.

19. Gordia AP, Quadros TMB, Campos W. Variáveis sociodemográficas como determinantes do domínio meio ambiente da qualidade de vida de adolescentes. Ciênc Saúde Coletiva. 2009;14(6):2261-8.

20. Interdonato GC, Greguol M. Qualidade de vida e prática habitual de atividade física em adolescentes com deficiência. Rev Bras Crescimento Desenvolv Hum. 2011;21(2):282-95.

21. Izutsu T, Tsutsumi A, Islam A, Matsuo Y, Yamada HS, Kurita H, Wakai S. Validity and reliability of the Bangla version of WHOQOLBREF on an adolescent population in Bangladesh. Qual Life Res. 2005;14(7):1783-9.
22. Castro ALB, Zamorano MAM. Calidad de Vida y Bienestar Subjetivo de Estudiantes Universitarios. Rev Psicologia Saúde. 2012;4(1):69-76.

23. Pires L, Rodrigues AM, Fisberg M, Costa RF, Schoen TH. Qualidade de vida de adolescentes modelos profissionais. Psic Teor e Pesq. 2012;28(1):71-6.

24. Canha LMN. Comportamentos e saúde nas crianças e adolescentes com deficiência motora. Rev Psicol Criança Adolescente. 2010;1(2):135-56.

25. Roehrs H, Maftum MA, Zagonel IPS. Adolescência na percepção de professores do ensino fundamental. Rev Esc Enferm USP. 2010;44(2):421-8.

26. Araújo CM, Oliveira MCSL. Significações sobre desenvolvimento humano e adolescência em um projeto socioeducativo. Educ Rev. 2010;26(3):169-94.

27. Al-Fayez GA, Ohaeri JU. Profile of subjective quality of life and its correlates in a nation-wide sample of high school students in an Arab setting using the WHOQOLBref. BMC Psychiatry. 2011;11(71):1-12.

28. Lin J-H, Ju Y-H, Lee S-J, Yang Y-H, Lo SK. Examining changes in self-perceived quality of life in children and adolescents with physical disability using a longitudinal design. Disabil Rehabil. 2011;33(19-20):1873-9.

29. Novaes RG, Trugillo EA. O aluno surdo no contexto do ensino regular. Rev Eventos Pedagógicos. 2011;2(2):210 -9.

30. Santos, LHC, Grisotto KP Rodrigues DCB, Bruck I. Inclusão escolar de crianças e adolescentes com paralisia cerebral: esta é uma realidade possível para todas elas em nossos dias? Rev Paul Pediatr. 2011;29(3):314-9.

31. Oliveira JBG. A perspectiva da inclusão escolar da pessoa com deficiência no Brasil: um estudo sobre as políticas públicas. Rev Tempos Espaços Educ. 2011;4(6):147-59.

\section{Endereço para correspondência:}

Christielle Lidianne Alencar Marinho

Avenida da Integração, 275 apto 12

Bairro: Centro

CEP: 56328-010 - Petrolina - PE - Brasil

E-mail: christiellealencar@yahoo.com.br 